\title{
SOLAPACIONES ENTRE COGNICIONES Y AFECTOS COMO COMPETENCIAS HUMANAS
}

\author{
Vicente Pelechano
}

Universidad de La Laguna (Tenerife)

\section{RESUMEN}

La división tripartita de los dominios psicológicos ha facilitado el estudio de áreas y fenómenos claros aunque ha soslayado aspectos importantes del funcionamiento psicológico humano. Se defiende que el sector de solapación entre afectos, conaciones y cogniciones resulta de especial interés y se enuncian los intentos más relevantes propuestos, con sus ventajas e inconvenientes. Se propone una opción teórica en la que se generen instrumentos de evaluación adecuados que recojan esa solapación y se integren los resultados dentro de un modelo en el que primen distintas racionalidades y un estudio dinámico de la afectividad. Las inteligencias múltiples se han acercado al problema aunque no lo han resuelto.

\footnotetext{
Palabras clave: AFECTIVIDAD-COGNICIÓN COMO POTENCIALIDADES HUMANAS, AFECTVIDAD POSITIVA Y EFICACIA, DINÁMICA AFECTVA Y COMPORTAMENTAL.
}

Este trabajo forma parte de un proyecto sobre sabidurla subvencionado por la Comisión Interministerial de Ciencia y Tecrologla. Las opiniones vertidas en este manuscrito en nada comprometen al mencionado organismo y son de exclusiva responsabilidad del autor.

Correspondencia: vpelecha @ull.es 


\section{SUMMARY}

The classical tripartite division in psychology (affective, conative and cognitive processes) has been useful through centuries; in the last decades the interface among these parts has become in a very important domain relevant for the study of social and human competence. Social intelligence, psychopathology, emotional intelligence, wisdom and practical intelligence have a lot of proposals and objectives common: to isolate social and human strengths directed to better human being. It is demanded a model in which interactive designs, multivariate orientation, non-reversibility, non-linearity, flexibility and some inconsistency play a important role. The proposal of multiple intelligences has some value but it is not the solution: different rationalities and the study of dynamic of affectivity is compulsory.

Key words: INTERFACE OF EMOTIONALITY AND COGNITION AS HUMAN STRENGTH, POSITIVE AFFECT, RATIONALITY AND EFFICACY.

\section{CONCEPCIÓN TRIPARTITA DE LOS FENÓMENOS PSICOLÓGICOS}

Tradicionalmente, el análisis de los fenómenos psicológicos ha seguido las líneas de una clasificación: cognición, afecto y conación (voluntad). Esta división tripartita que ya se encuentra, hace unos siglos, en Tetens, maestro de Kant (con antecedentes en la Grecia clásica), se ha mantenido hasta nuestros días. La cognición recogería los elementos comprometidos fundamentalmente con las aptitudes y "competencias", el afecto los aspectos emocionales y la conación (o elementos conativos y proactivos de la conducta) en una suerte de solución de compromiso en la que se encontraban presentes elementos de los dos campos anteriores. De una u otra forma, la cognición humana ha capitaneado el desarrollo del estudio de las aptitudes, capacidades y/o competencias; la conación, el estudio de los motivos conscientes y voluntad humana y la emoción, por su parte se ha ido quedando restringida a elementos "complementarios" que daban color o tono a los otros dos dominios pero que apenas tenían entidad por sí mismos, que no fuera "perturbar" o, en algún caso, 
"facilitar" la eficacia comportamental (generación de expectativas de éxito como elemento a mitad de camino entre lo cognitivo, lo afectivo y lo motivacional). La "psicología de los afectos" se ha desarrollado independientemente de la psicología de la personalidad y se ha visto reducida a elementos situacionales y poco estables a lo largo de situaciones y tiempos distintos, en una discutible reconversión de la emocionalidad al estudio de la "emoción".

Durante buena parte del siglo $X X$ el estudio psicológico de la afectividad se encontraba restringido a sus aspectos básica aunque no exclusivamente negativos conceptualizados como activación (relacionado con psicofisiologia, ansiedad, nivel impulsivo y aprendizaje) o como un mecanismo de cambio comportamental en el caso de interrupción brusca del foco atencional debido a una redirección de la atención producida por la aparición de estimulación nueva, fuerte o intensa (Isen y Hastorf, 1982; Simon, 1967).

Las competencias humanas eran conceptualizadas como los elementos comprometidos con la eficacia comportamental y sus determinantes "cognitivos" con inclusión, hacia la mitad de siglo XX de la motivación, aunque su peso ha sido indudablemente menor (los aspectos afectivos y los motores eran ignorados). Esta situación se ha ido modificando a medida que se ha acrecentado la investigación en emoción-afectividad (los aspectos psicomotores van un tanto retrasados, Rosenbaum, 2005).

\section{SUGERENCIAS ALTERNATIVAS ACERCA DE LAAFECTIVIDAD}

En este trabajo se propone que la afectividad (o una buena parte de ella) representa una fuente competencial promotora de supervivencia y de eficacia comportamental. Y ello desde una perspectiva en la que la afectividad representa uno de los sustratos filogenéticos de la evolución (posibilitante de la reproducción de la especie), acompañante de todos los fenómenos psicológicos y elemento clave para entender el continuo de eficacia-ineficacia comportamental desde un análisis intraindividual. En la medida en que esto sea así, los procesos cognitivos podrían ser cualificados no solamente con respecto al dominio en el que se desarrollan y actúan sino, también, por el tipo de relación que poseen con la afectividad. 
Desde aquí, la división de la afectividad en "positiva" y "negativa" supone un primer acercamiento, aunque muy elemental, a los factores y procesos afectivos. Se supone que un afecto "positivo" será siempre positivo y uno negativo lo será siempre negativo, aunque la cualificación de "positivo" y "negativo" no está muy clara (el amor es un afecto positivo y el odio, negativo, aunque el calificativo no corresponde a su valor adaptativo o supervivencial y la dinámica de los afectos puede convertir uno en otro en la medida en que no existen los afectos en abstracto sino encarnados en personas $y$ afectos positivos $y$ negativos pueden darse a la vez y respecto a una misma persona $u$ objeto; o pasar con rapidez de uno a otro. En más de una situación, se odia y se ama, a la vez, sin que ello sea síntoma, ni marcador, de psicopatología ${ }^{1}$ ).

Algunas líneas discursivas que pueden resultar de interés en el presente contexto son las siguientes:

- Por lo que se refiere al estado funcional cotidiano, la afectividad acompaña aunque no de forma consciente debido a la habituación (automatización). Y, usualmente el afecto es "positivo" de bienestar dentro de unos márgenes determinados. Cuando se pasan estos límites el afecto "se hace consciente" y los cambios, no especialmente intensos, son estudiados en laboratorio.

- La intensidad que presentan los afectos es, al margen de los contenidos afectivos (y dentro de cada uno de ellos), una fuente de diferencias individuales. Y se asume que es una característica psicológica identificadora de las personas, que es relativamente estable a lo largo de toda la vida de las personas y no identificable con impulsividad o inestabilidad emocional.

- Si la intensidad de los afectos es grande, se promueve una disminución en la capacidad de análisis de la propia realidad, aunque la distorsión puede llevar a un incremento en la eficacia de una parte de la conducta.

- En la realización de tareas, la afectividad se encuentra asimismo presente y facilita o perturba el rendimiento de las mismas. La manera

\footnotetext{
'Algo similar hay que decir respecto a algunas emociones que, como la ira, son consideradas "negativas" en algunos contextos cotidianos occidentales pero no en otros. Y su carencia, en más de una ocasión, posee connotaciones negativas. La utilización del desdén en las relaciones de cortejo humano no siempre significa devaluación de la persona (recuérdese El desdén can el desdén de Lope de Vega.
} 
de apresar estas influencias es con análisis intraindividual y no interindividual (la variabilidad de rendimiento por influencia del afecto se hace patente cuando se observa el rendimiento de un individuo, en la medida en que las interacciones son individuales).

- El estudio de la afectividad en un plano interindividual y evolutivo sugiere que: (a) los distintos parámetros de la afectividad (contenido, frecuencia, duración, intensidad, cambio de un afecto a otro) son otras tantas fuentes de diferencias individuales; (b) el impacto de la afectividad sobre la conducta observable es más importante en los primeros estadios del desarrollo humano (primera y segunda infancia) y en los últimos. Asimismo existen "crisis" que van acompañadas de desajustes bioquímicos con gran impacto de la afectividad sobre la conducta (la adolescencia en ambos sexos y también durante la menopausia en las mujeres).

- Tanto o más que el contenido concreto de los afectos en una persona importan los parámetros de intensidad, fluctuación y duración, sin los cuales el estudio de los afectos se convierte en un dominio muy difícil de integrar en la taxonomía comportamental. El estudio de la "transformación" de emociones en afectos más estables (sentimientos, Lucas, Diener y Larsen, 2003) y complejos (bienestar subjetivo, Keyes y Magyar-Moe, 2003) no depende solamente del paso del tiempo.

- Si bien se ha dedicado esfuerzo al estudio de la base bioquimica de la afectividad, el estudio de las interacciones y dinámica entre bioquímica y conducta no posee un estado lo suficientemente satisfactorio como para que exista un modelo comprensivo en el que los afectos positivos, los afectos negativos, su dinámica y parámetros de intensidad y duración así como la psicopatologia de los trastomos afectivos se encuentre explicada. La apelación a la dopamina (para el afecto positivo), por un lado y a la noradrenalina y la serotonina (para el afecto negativo), aunque representan un avance, son radicalmente insuficientes para explicar la dinámica interactiva que define la vida afectiva, y no representan más que acercamientos bastante burdos para la comprensión de la vida afectiva.

- El papel que juega la afectividad y los cambios moderados de afectividad en la realización de tareas "cognitivas" es muy distinto en función de las tareas a realizar. El papel de la afectividad en las ciencias 
sociales (y en la solución de los problemas que en éstas se encuentran planteados) tiende a ser más claramente observable y su compromiso con el éxito en las tareas es mayor que en las ciencias naturales (por ejemplo, el valor de la empatía y de la comprensión de los demás en las ciencias sociales). En todo caso, podríarı llegar a equilibrarse en ambos dominios en niveles muy superiores y cuando los humanos llegan a un claro estado de excelencia, lo que no es nada frecuente (cuando llegan a convertirse en sabios sobre las cuestiones básicas de la vida, lo que no necesariamente lleva consigo un único modelo funcional).

- En el caso de fenómenos humanos muy complejos, como la psicología de la sabiduría, la afectividad ocupa un lugar relevante aunque difícil de operacionalizar. Por una parte debe reconocerse la presencia de aspectos de afectividad positiva, aunque esta presencia no debe ser tan intensa que impida el pensamiento o la acción (como sucede en el éxtasis místico); por otra, debe estar ausente psicopatología de la afectividad (que puede encontrarse presente en la genialidad y la superdotación intelectual) y su presencia, aunque notada, no debería ser especialmente intensa en sus manifestaciones.

- Una posible consecuencia de lo anterior es que la interacción entre afecto y cognición en los conocimientos de las ciencias sociales y, más en especial en aquellos dominios relacionados con la interacción personal promovería una forma de conocer y de discurrir un tanto distinta de la propia de las ciencias naturales, y en ella, el impacto de afectos y cogniciones provenientes de contextos de vida, experiencia personal y lecturas "no-científicas" ocuparía un lugar especial, incluso, en científicos que ofrezcan modelos y resultados "científicos": el papel de las experiencias personales y en suma, de conocimientos "legos" se puede rastrear en todo trabajo cientifico-psicológico que se ocupe de temas de interés "aparente".

- Finalmente, para no alargar más estas consideraciones, el papel que desempeñaría la afectividad en las distintas acepciones de sabiduría (lega, interpersonal, intrapersonal, teorética, decantación de experiencia profesional, socio-política) podría ser distinta aunque en todos los casos debería ser afectividad positiva y nunca extremada en intensidad y no exigitiva de niveles funcionales muy altos o de excelencia para con los demás. 


\section{UN CAMINO SIMPLE: EMOCIÓN Y EMOCIONALIDAD EN EL DICCIONARIO DE LA REALACADEMIA ESPAÑOLA}

Una de las soluciones ante la complejidad de problemas e interacciones psicológicas sería apelar a las significaciones "de diccionario" como un punto de partida. Cuando se cae en la tentación de acudir al de la Real Academia Española de la Lengua se encuentran una selección de expresiones cuyo resumen de significado sería el siguiente:

- Emoción: alteración del ánimo intensa y pasajera, agradable o penosa, que va acompañada de cierta conmoción somática. Asimismo, se recoge la acepción de emoción como interés expectante con que se participa en algo que está ocurriendo. La significación de emocionalidad se zanja remitiendo a "emocional" y éste, a ser afectado por las emociones.

- Pasión: se recogen varias significaciones de contenido psicológico: (a) acción de padecer; (b) lo contrario a la acción; (c) perturbación o afecto desordenado del ánimo; (d) inclinación o preferencia muy vivas de alguien a otra persona; (e) apetito o acción vehemente a algo; (f) de ánimo: tristeza de pasión, abatimiento, desconsuelo. Dos expresiones de una misma idea, "compasión" y "simpatía", han tenido desarrollos distintos y se han diferenciado de la "empatía" incluso dentro de la psicología clínica.

- Afecto: cada una de las pasiones del ánimo, como la ira, el amor, el odio, etc. y especialmente el amor o el cariño.

- Afectividad. (a) desarrollo de la propensión a querer; (b) conjunto de sentimientos, emociones y pasiones de una persona; (c) tendencia a la reacción afectiva o sentimental.

- Estado de ánimo: disposición en que se encuentra alguien, causada por la alegría, la tristeza, el abatimiento, etc.

- Sentimiento: (a) acción y efecto de sentir o sentirse; (b) estado afectivo del ánimo producido por causas que lo impresionan vivamente; (c) estado del ánimo afligido por un suceso triste o doloroso.

No deja de resultar curioso que mientras el sentimiento y el afecto se relacionan con lo que tradicionalmente se entiende en psicología como "afectividad" (aunque sin muchas especificaciones posteriores), la expresión de "sentir" posee en español connotaciones cognitivas 
indudables como en las siguientes significaciones: oír o percibir con el sentido del oído, experimentar una impresión, placer o dolor corporal o espiritual, juzgar, opinar, formar parecer o dictamen (como en la expresión "digo lo que siento") y presentir o barruntar lo que ha de sobrevenir. $Y$ algo en la misma línea sucede en el caso del ánimo que se define de manera variada como alma o espíritu en cuanto es principio de la actividad humana, como valor, esfuerzo o energía que lo acercaría a elementos conativos de la acción, como sucede con la acepción de intención o voluntad; a la vez se define como atención o pensamiento (cognición).

En suma: la emoción es situacional, la afectividad se refiere en general a referentes situacionales (como la emoción) y otros que no lo son tanto (como parece desprenderse de sentimientos y pasiones). Además, las significaciones de las expresiones que cabría esperar se relacionarían con afectividad, se encuentran entreveradas con emocionalidad, motivación y cognición por lo que la diferenciación "psicológica" tripartita, en español, se encuentra poblada por un conjunto de conceptos "difusos" entre las tres ramas de "procesos psicológicos". En todo caso, la significación connotativa de las expresiones no aparece cargada tan solo de elementos negativos, sino que recogen aspectos positivos (amor) y negativos (odio), y no existen suposiciones acerca de correlatos sobre eficacia comportamental relacionada con ellos.

En cuanto a propuesta terminológica la emoción y el afecto se referirían a elementos situacionales; el ánimo a fenómenos contextuales de duración intermedia, mientras que sentimiento podría referirse a aspectos más consistentes y estables de la conducta, de corte más básico y resistentes al cambio.

\section{UN MÚLTIPLE ARRANQUE HISTÓRICO}

Los cambios sociales que arrancaron en los 60 del pasado siglo promovieron la aparición de modos alternativos de pensar en la afectividad humana y su relación con las competencias humanas. Entre las líneas de pensamiento que deberian ser tomadas en consideración se encuentran las siguientes:

1. La psicopatología: los trastornos mentales como problemas irresolutos con un gran peso de la emocionalidad yla interacción social. 
Con el desarrollo de la psicología del ego Jahoda $(1953,1958)$ sugería que la psicopatología podía entenderse como una carencia en las capacidades de solución de problemas intra-e interpersonales. Entre estas capacidades se encontraba la necesidad por lograr el manejo de las emociones propias y ajenas, en la medida en que las emociones ayudaban a distorsionar las percepciones e ideas correctas de los sucesos y personas. Desde los comienzos de la terapia de conducta se apeló a la seguridad personal (la asertividad) que implicaba una emocionalidad de confianza y seguridad en sí mismo/a como elementos centrales en la eliminación de trastornos de ansiedad (recuérdese a Wolpe, por ejemplo). Esta apertura hacia el análisis de las interacciones personales ayudó a generar programas de manejo y control de la emocionalidad y de la impulsividad como elementos clave en el tratamiento de psicopatías y otros problemas de ansiedad y depresión. Repárese que en estos análisis, procedentes de escuelas dispares, la emocionalidad posee componentes "positivos" (aserción personal) y "negativos" (las disfunciones emocionales y problemas en relaciones interpersonales como causa de psicopatología) y los objetivos que se perseguían eran el manejo y control de la emoción, 0 en su caso, la implantación de "sentimientos positivos" (tranquilidad, sosiego, serenidad, seguridad) como antitéticos de los sentimientos negativos (tristeza, sufrimiento, ansiedad). En nuestros días, la aceptación de la emocionalidad, así como su manejo y control, forma parte integrante de la mayoría de opciones terapéuticas "cognitivas" o "conductual-cognitivas".

2. La reconceptualización del valor de la emocionalidad como fuerza evolucionista. Resulta cuanto menos extraño el tratamiento relativamente escaso de la emocionalidad humana en psicología cuando ya Darwin subrayó la importancia de la emoción para la supervivencia de las especies. Emocionalidad y reconocimiento de la existencia de los demás (con colaboración) formarian la base de la supervivencia como especie humana.

La emocionalidad posee una naturaleza bifronte: por una parte se trata de un fenómeno que se da en el individuo y, por otra, posee una dinámica básicamente interpersonal (Berscheid y Reis, 1998, Reis, Collins y Berscheid, 2000, Zajonc, 1998). La existencia de procesos básicos de carácter emocional en los primeros años de vida como el 
"apego", entendido como una tendencia evolucionada para que un individuo mantenga la proximidad física y psicológica de otro ser humano más poderoso y capaz de cuidar de él (Bowlby, 1969/1982); apego que, al parecer, influye en las relaciones afectivas que establecen los humanos cuando llegan a adultos (Shaver y Hazan, 1993).

El establecimiento de lazos emocionales con los demás se ha caracterizado como la mayor fuerza con que ha contado la especie humana para conformarse como es en la actualidad (Berscheid, 2003) $y$, por lo tanto formaría parte esencial de la naturaleza humana de manera tanto o más fundamental que la "racionalidad" (filogenéticamente, al menos, es anterior).

3. El impacto comportamental-cognitivo de la emocionalidad positiva. Desde una perspectiva teóricamente más restringida, Alice $M$. Isen $(200,2002,2003)$ ha postulado que deberían ofrecerse modelos integrados de funcionamiento emocional, cognitivo y motivacional. De hecho, el afecto es una parte sustantiva de los procesos de pensamiento y motivacionales, tanto de los que promueven una eficacia comportamental como de los que la dificultan. La propuesta de Barbara L. Fredrickson sobre afectividad positiva (la teoría de ampliación y construcción) defiende que frente a los efectos específicos y restringidos de la afectividad negativa, la positiva promueve una amplificación de estrategias cognitivas y efectos a largo plazo que, en último término, se encuentran anclados en la filogénesis de la conducta humana (Fredrickson, 2001; Fredrickson y Losada, 2005), su puesta a prueba debe acudir a modelos de relaciones no lineales y la manera eficaz de estudiar el papel del afecto en la conducta humana consiste en puntuaciones derivadas de las relaciones y equilibrios entre afectividad positiva y negativa. En la medida en que se ha dado un peso mayor al papel que desempeña la afectividad negativa, señalamos algunas líneas de investigación con resultados que son representativos de la facilitación emocional de la conducta eficaz:

- Solución creativa de problemas en organizaciones.

- Facilitación de relaciones en negociaciones complejas.

- Los afectos positivos facilitan la búsqueda de soluciones alternativas

- Afectos positivos promueven ayuda razonable, generosidad y responsabilidad social.

- Afectos positivos potencian mantenimiento de tareas que se están 
realizando aunque no impiden darse cuenta de informaciones acerca de aspectos negativos o fracasos parciales que estén sucediendo.

- Los efectos de la afectividad sobre la conducta dependen tanto del tipo o contenido de afecto como de su intensidad. Con afectos muy intensos los efectos sobre la acción son más claros, favoreciendo o interfiriendo el curso de la acción y las razones para la misma acción.

- Uno de los resultados obtenidos con claridad es que se han logrado efectos a medio plazo con inducciones de afectos positivos durante períodos de tiempo cortos. $Y$ se han obtenido efectos similares con inducción de afectos disposicionales y afectos situacionales (Aspinwall \& Richter, 1999; Estrada \& al., 1997; Staw \& Barsade, 1993).

Todo ello sugiere que la afectividad representa un conjunto de capacidades psicológicas de gran potencialidad y de las que apenas se ha desarrollado su estudio.

4. Las "inteligencias múltiples" y su conexión con la interacción social y las emociones. Una anotación sobre la "inteligencia emocional".- Ya hace casi un siglo, Thorndike denunciaba que los tests de inteligencia al uso adolecían de un problema grave: prescindían de la evaluación de la competencia social, problema que no se ha resuelto satisfactoriamente hasta nuestros días, aunque se han llevado a cabo distintos intentos entre los que hay que anotar distintas propuestas sobre inteligencias múltiples (Gardner, 1983, 1993; Pelechano, 1989) en las que se reconceptualiza la significación de las competencias humanas (la "inteligencia" sería una de ellas) y se proponen otras. Otra opción que ha tenido una gran difusión (por la versión periodística de Goleman, 1995) es la propuesta de Salovey y Mayer (1990) de la "inteligencia emocional".

Desde una perspectiva antropológica y psicosocial, las propuestas de las "inteligencias múltiples" representan una manera de entender las competencias humanas en las que la emocionalidad forma parte sustantiva de una buena parte de ellas. Las llamadas "inteligencias sociales" y las "inteligencias intrapersonales" incorporan el conocimiento de los propios estados emocionales y los de los demás en un intento por estudiar las situaciones y problemas de la vida desde una perspectiva alejada del discurso dicotómico (verdadero/falso, bueno/malo) y con la utilización de una lógica polivalente en la que el realismo, la eficacia, la necesidad de tomar en cuenta las opiniones y 
afectos de los demás y la reinterpretación de la "racionalidad humana" como la propia de un depredador, forman parte sustantiva. Se asume que los procesos psicológicos comprometidos en la percepción y manejo de las situaciones interpersonales resultan distintos de los comprometidos en el análisis y tratamiento de los problemas matemáticos. Y las aplicaciones que se han llevado a cabo por lo que se refiere a las habilidades interpersonales van desde el tratamiento de casos clínicos, hasta los cambios en actitudes que promuevan una convivencia más apacible y tolerante en un rango de edad que va desde los 4 hasta los 93 años (Pelechano, 1991,1996). Las dimensiones que poblarían este mundo de competencias interpersonales van desde estilos de afrontamiento de situaciones complejas de vida (como puede ser enfrentarse a una enfermedad propia o de alguien cercano), pasando por la aserción personal y otras dimensiones de inteligencia interpersonal como la asunción de posiciones distintas en un conflicto, la percepción de los sentimientos propios y de los demás, la previsión de consecuencias inmediatas y mediatas de acciones propias o ajenas, la segmentalización de los problemas complejos en otros más simples, la atribución de causas a las acciones y hechos que nos rodean y la búsqueda de distintas opciones de solución ante un mismo problema (Pelechano, 2000).

La inteligencia emocional defendida por Salovey y Mayer a lo largo de más de una década estaría compuesta por cuatro grandes dimensiones teóricas: (a) percepción de emociones (capacidad para identificar las emociones propias y las ajenas, así como las que producen historias, música, arte o ciertos objetos); (b) utilización de la emoción para facilitar el pensamiento (capacidad para generar, utilizar y sentir emoción como un elemento necesario para comunicar sentimientos o utilizarlos en otros procesos psicológicos); (c) comprensión de las emociones (capacidad para comprender la información emocional, su combinación y progreso en la comprensión de las transiciones en las relaciones) y (d) manejo de emociones (capacidad para mantenerse abierto/a a las emociones y a regularlas en la acción intra e interpersonal). Los autores que han acuñado la expresión han ofrecido, además, dos baterías de pruebas (la Multifactor Emotional Intelligence Scale, MEIS de Mayer, Caruso y Salovey, 1999 y el Mayer-Salovey-Caruso Emotional Intelligence Test, MSCEIT de 
Mayer, Salovey y Caruso, 2001, 2002). Los resultados correspondientes a consistencia interna de ambas baterias parecen satisfactorios, los correspondientes a validez diferencial con inteligencia tradicional también, aunque los resultados correspondientes a los correlatos comportamentales de las escalas son más bien pobres y escasos (este hecho no está claro que se deba únicamente a los problemas de elaboración de las pruebas sino a la propia conceptualización de algunas de estas dimensiones y su operacionalización).

5. Las renovadas y nunca terminadas "evoluciones" $y$ "refundiciones" de la psicología cognitiva: cognición y "racionalidad".Pasada la segunda mitad del siglo $X X$ apareció como un rótulo innovador y con mucha fuerza la etiqueta de "revolución cognitiva" como una alternativa a los conductismos imperantes. Esta "revolución" se ha repetido en diversas ocasiones hasta ir agotando su fuerza revolucionaria. De todas las versiones, posiblemente la que ha gozado de mayor desarrollo y reconocimiento de "ciencia dura" ha sido la que ha utilizado la metáfora del ordenador: la cogrición humana se volvió "procesamiento humano de información" y, cuando esa información se restringió a la de los procesos e interacciones humanas, el modo de pensar humano se entendió como el de las operaciones de un ordenador (aunque un poco lento y con fuertes restricciones de memoria de trabajo y de almacenamiento); y lo que no seguía las sacrosantas leyes del software... no era humano. Y ello, por supuesto, no ayudó al análisis de situaciones humanas complejas y se "impuso" una "racionalidad" humana que podía funcionar en la solución de problemas matemáticos pero no psicológicos en los que hubiera comprometidos sentimientos (ien dónde no los hay?). Tuvo que pasar alguna otra revolución para aceptar que ni la ciencia es tan racional, ni lo es el ser humano ni, posiblemente lo que sea más importante, existe una única manera de "ser racional" y que frente al isomorfismo y reduccionismo de los procesos que estudiaba la psicología "cognitiva", se debía ampliar tanto el número como el tipo de procesos psicológicos relevantes, así como de las interacciones entre los diversos sistemas funcionales, de estudio imposible desde una perspectiva "cognitiva" de laboratorio. El estudio de la irracionalidad humana no se explica solamente por la inclusión de emociones (ni siquiera ello explicaría la 
racionalidad) sino por el propio decurso "cognitivo" cuando éste debe ocuparse de los problemas complejos que presentan los humanos.

$Y$ todo ello ha propiciado la gestación de unas corrientes en psicología en las que se defiende que para la comprensión del ser humano deben incluirse distintos tipos de racionalidades, así como de emocionalidades y sentimientos. La integración de todas estas corrientes se encuentra todavía lejos de ser una realidad inmediata, aunque los estudios encaminados a esta unificación son perentorios en la medida en que se siga defendiendo que la psicologia es una ciencia y que se ocupa, también de problemas individual, interpersonal y socialmente relevantes, más allá de los tiempos de reacción y de las series de fonemas y silabas con y sin sentido. Y no aceptar, sin más, la esquizoidia presente en ciertos autores contemporáneos que, tras una dilatada vida de dedicación a causas "naturalistas", están aceptando una división clara entre la ciencia y sus propios problemas y temas de interés (cfr. por ejemplo, Kendler, 2005).

\section{LA APELACIÓN A LA CONDUCTA OBSERVABLE Y LA ¿BASE BIOQUIMICA DE LAAFECTIVIDAD?}

La investigación en estos conceptos ha sido muy abundante y de calidad. El problema es que a la hora de estudiar "relaciones" e "interacciones" el esquema ha sido en todos los casos el siguiente: estudio de una tarea a realizar (denominada "cognitiva" usualmente), promoción o inducción de una afectividad dada (positiva o negativa) de corte situacional y que ha sido distinta para distintos grupos experimentales (o experimental y control) y análisis de las diferencias en resultados. El criterio, como cabría esperar, tenía que ver con la "conducta eficaz" que se analiza en contextos distintos (solución de problemas, realización de tareas de grupo, aprendizaje, conducta en organizaciones, etc.) y los resultados obtenidos tienden a ser coherentes al margen de la forma de inducción del afecto, y del tipo de tarea propuesta en solución de problemas -usualmente impersonales-, con implicaciones para una teoría bioquímica del afecto positivo basado en el metabolismo de la dopamina (Ashby, Isen y Turken, 1999).

Es posible que el tipo de tarea a realizar o situación con la que se enfrenta el ser humano sea irrelevante, aunque no es muy probable. 
Preocupada como está la investigación en la conducta eficaz y observable por parte de los demás con criterios objetivos, la mayor parte del trabajo realizado se ha referido a la "solución" de problemas $y$ "rendimiento" eficaz en tareas objetivamente delimitadas (aunque no especialmente relevantes ni vitales para el funcionamiento humano) y en estos contextos parece que la afectividad positiva es favorecedora de la eficacia en la solución de estos problemas que, presumiblemente, son "realizados" por procesos cognitivos y que parecen ser concomitantes con la acción de la dopamina (en situaciones y personas "normales", porque cuando se trata de personas con trastornos graves como depresión, el asunto es otro). Sin embargo, cuando las tareas o problemas son muy importantes, o se trata de la evaluación de sentimientos complejos (como el amor de pareja, la felicidad o el "sentirse bien") el volumen de información es mucho menor y los resultados menos claros. $Y$ todo ello puede interpretarse desde un punto de vista alternativo defendido por el modelo de parámetros: la calificación de los tipos de emociones no resulta satisfactorio apelando tan solo a los contenidos afectivos, sino que resultan tanto o más importantes otros parámetros como la intensidad, duración y frecuencia de la emoción. Así, la intensidad es la que "cualificaría" una emoción en positiva o negativa en función de la situación vital de que se trate. Una "emoción positiva" de una misma intensidad puede dejar de serlo por habituación a la misma y se puede convertir en negativa o en "irrelevante" (estado muy cercano a la anhedonia específica de esa emoción); al contrario una afectividad negativa se puede "transformar" en positiva si cambia el contexto de interpretación. Una misma ciudad puede ser "percibida" de maneras distintas en función del estado de ánimo que se tenga. $Y$ existen diferencias individuales estables en todos estos fenómenos a los que acabamos de hacer referencia.

Con el fin de sugerir puntos de referencia y de análisis crítico proponemos las siguientes generalizaciones:

(a). La afectividad se encuentra presente en toda acción humana y forma parte del equipamiento filogenético básico de cornpetencias humanas. Usualmente el ser humano posee un tono afectivo positivo que acompaña a las actividades usuales. Cuando cambia el tipo, intensidad o duración de la actividad, más allá de un umbral de tolerancia que es específico para cada ser humano o tipo de ser 
humano, la interacción entre afectividad, la cognición y la conducta se modifica sustancialmente.

(b). La agrupación de los afectos en dos categorías, positivos y negativos, tiene un valor contextual y situacional, cuya estructura y dinámica es compleja y depende de otros parámetros $y / 0$ características emocionales y del contexto como la intensidad, la duración y la sensibilidad al cambio emocional.

(c). Es posible diferenciar la afectividad (positiva y negativa) de poca o escasa intensidad, del nivel de activación. Esta diferencia posee correlatos comportamentales distintos.

(d). El aumento de dificultad o gravedad de las situaciones de vida lleva consigo un cambio no solo en la estructura sino también en la dinámica de las emociones. Emociones que interactúan con las situaciones de vida de manera continua.

(e). La interacción entre lo cognitivo y lo emocional es muy compleja $y$, dentro de grandes márgenes, un aspecto puede modificar al otro (facilitando o dificultando el rendimiento). Si un problema no tiene solución claramente identificable, se apela a la modificación emocional. Si la emocionalidad es muy intensa y prologada, se puede actuar tanto en la orientación de solución de los problemas planteados, como en la del recambio de emociones. La propuesta lega de que una solución eficaz modifica los sentimientos no parece funcionar, ni siempre, ni en todos los casos, en los adultos que viven en nuestra sociedad.

(f). Entre ser eficaz y "sentirse bien", la sociedad promueve lo primero, mientras que lo segundo tiende a ser importante socialmente en las primeras fases de la vida y en las últimas (cuando se trata de seres humanos "no productivos"). Para el resto de la vida prima la eficacia sobre la afectividad positiva y esta eficacia se entiende como una promoción de lo cognitivo y aptitudinal.

\section{CONCLUSIONES}

La irrupción del estudio científico de la afectividad en la psicología ha promovido cambios en conceptualización (no representa una limitación a la acción eficaz sino un conjunto de competencias) y, en los últimos tiempos se ha sugerido la expresión de "fuerzas" o potenciaciones del ser humano. 
Al reconocimiento del valor de la afectividad en esta nueva orientación ha ayudado la aparición de estudios en los que se ha subrayado la importancia de los elementos "positivos" de la psicología humana, así como una crítica y ampliación de la idea de "cognición", que debe entenderse en plural, como racionalidad y discurso científico.

El análisis bioquímico puede iluminar algunas relaciones e interacciones entre afectos y cogniciones, aunque en la actualidad aportan solamente retazos de lo que sin duda es una imagen mucho más compleja y rica.

Los estudios sobre los elementos cognitivos psicosociales y positivos han ayudado y ayudan a situar el mundo afectivo en un lugar de mayor peso dentro de la psicología que el que tenía hasta hace unos años. $Y$ ayuda, definitivamente a ampliar y perfilar otras acepciones de racionalidad frente a las dicotómicas categoriales y excluyentes que han caracterizado la investigación sobre cognición humana en casi el último medio siglo.

Con todo ello, finalmente, es muy posible que se ofrezca una imagen general mucho más cercana a la realidad de la riqueza psicológica del ser humano a comienzos del siglo XXI.

\section{REFERENCIAS BIBLIOGRÁFICAS}

Ashby, F. G, Isen, A. M. \& Turken, U. (1999).- A neuropsychological theory oif positive affect and its influence on cognition, Psychological Review, 106, 529-550.

Aspinwall , L. G \& Richter, L. (1999).- Optimism and self-mastery predict more rapid disengagement from unsolvable tasks in the presence of alternatives, Motivation and Emotion,23, 221-245.

Bersheid, E. (2003),- The human's greatest strength: Other humans,

En L. G Aspinwall \& U. M. Staudinger (eds).- A psychology of human strengths. Fundamental questions and future directions for a positive psychology, 37-48, Washington, American Psychological Association. Bersheid, E. \& Reis, H. T. (1998).- Attraction and close relationships.

En D. T. Gilbert, S. T. fiske \& G Lindzey (eds).- The handbook of social psychology, vol.2, 193-281.

Bowlby, J. (1982).- Attachment and loss: Vol. 1. Attachment, (2 ${ }^{\text {nd }}$ edit.), New York, Basic Books (orig. 1969). 
Estrada, C. A., Isen, A. M. \& Young, M. J. (1994).- Positive affect influences creative problem solving and reported source of practice satisfaction in physicians, Motivation and Emotion, 18, 285-299.

Estrada, C. A., Isen, A. M. \& Young, M. J. (1997).- Positive affect facilitates integration of information and decreases anchoring in reasoning among physicians, Organizational and Human Decision Processes, 72, 117-135.

Fredrickson, B. L. (2001).- The role of positive emotions in positive psychology: The broaden-and-build theory of positive emotions, American Psychologist, 56, 218-226.

Fredrickson, B. L. \& Losada, M. L. (2005).- Positive affect and the complex dynamics of human flourishing, American Psychologist, 60, 678-686.

Gardner,H.(1983).- Frames of mind. The theory of multiple intelligences, New York, Basic books.

Gardner, H. (1993). - Multiple intelligences. The theory in practice, New York, Basic Books.

Goleman, D. (1995).- Emotional intelligence,New York, Bantam. Isen, A. M. (2000).- Positive affect and decision making. En M. Lewis \& J. M. Haviland-Jones (ed).- Handbook of emotions, $2^{\text {nd }}$ edit., 417435, New York, Guilford Press.

isen, A. M. (2002).- A role of neuropsychology in understanding the facilitating influence of positive affecton social behavior and cognitive processes. En C. R. Snyder \& J. S. Lopez (eds).- Handbook of positive psychology, 528-540, New York, Oxford University Press. Isen, A. M. (2003).- Positive affect as a source of human strength. En

L. G. Aspinwall \& U. M. Staudinger (eds).- A psychology of human strengths. Fundamental questions and future directions for a positive psychology, 179-195, Washington, American Psychological Association.

Isen, A. M. \& Hastorf, A. H. (1982).- Some perspectives on cognitive social psychology. En A. H. Hastorf \& A. M. Isen (eds).- Cognitive social psychology, 1-31, New York, Elsevier North-Holland.

Jahoda, M. (1953).- The meaning of psychological health, Psychological Casework, 34,349-354.

Jahoda, M. (1958).- Current concepts of positive mental health, New York, Basic Books. 
Kendler, H. H. (2005).- Psychology and phenomenology: Aclarification, American Psychologist,60, 318-324.

Keyes, C. L. M. \& Magyar-Moe, J. L. (2003).- The measurement and utility of adult subjective well-being, 411-426. En S. J. Lopez \& C. R. Snyder (eds).-Positive psychological assessment, Washington, American Psychological Association.

Lucas, R. E., Diener, E. \& Larsen, R. J. (2003).- Measuring positive emotions, pp. 210-218. En S. J. Lopez \& C. R. Snyder (eds).Positive psychological assessment, Washington, American Psychological Association.

Mayer, J. D., Caruso, D. \& Salovey, P. (1999).- Emotional intelligence meets traditional standards for an intelligence, intelligence,27, 267-298.

Mayer, J.D., Salovey, P. \& Caruso, D. (2001).- The Mayer-Salovey-Caruso Emotional Intelligence Test (MSCEIT), Toronto, Multi-Health Systems.

Mayer, J. D., Salovey, P. \& Caruso, D. (2002).- Test Manual for the MSCEIT V.2, Toronto, Multi-Health Systems.

Pelechano, V. (dir)(1991).- Habilidades interpersonales en ancianos. Conceptualización y evaluación, Valencia, Alfaplús.

Pelechano, V. (dir)(1996).- Habilidades interpersonales. Teoria minima y programas de entrenamiento, vols, 1, 2 y 3, Valencia, Promolibro. Pelechano, V. (2000).- Psicología sistemática de la personalidad, Barcelona, Ariel.

Reis, H. T., Collins, W. A. \& Berscheid, E. (2000).- The relationship context of human behavior and development, Psychological Bulletin, $126,844-872$.

Rosenbaum, D. A. (2005).- The Cinderella of psychology. The neglect of motor control in the science of mental life and behaviour, American Psychologist, 60, 308-317.

Shaver, P. R. y Hazan, C. (1993).- Adult romantic attachment: Theory and evidence. En W. H. Jones \& D. Perlman (eds.).- Advances in personal relationships, vol. 4, 29-70., London, Jessica Kingsley.

Straw, B. M. \& Barsade, S. G. (1993).- Affect and managerial performance: A test of the sadder-but-wiser vs. happier-and-smarter hypotheses, Administrative Science Quarterly, 38, 304-331.

Zajonc, R. B. (1998).- Emotions. En D. T. Gilbert, S. T. Fiske \& G. Lindzey (eds).- The handbook of social psychology, $4^{\text {th }}$ edit., 591-632, New York, McGraw-Hill. 